\title{
NON-TYCHONOFF $e$-COMPACTIFIABLE SPACES
}

\author{
K. P. HART AND J. VERMEER
}

\begin{abstract}
We construct a non-Tychonoff space $X$ which is e-compactifiable, thus answering a question of S. Hechler. We also answer a question of R. M. Stephenson: whether there exists a Tychonoff space, the largest $e$-compactification of which has a noncompact semiregularization.
\end{abstract}

1. Introduction. All spaces are Hausdorff. In [He] S. Hechler introduced the class of $e$-compactifiable spaces, i.e. spaces which admit an $e$-compactification. He posed the question whether there exist non-Tychonoff $e$-compactifiable spaces. We show that such spaces exist. In [St] R. M. Stephenson observed that an e-compactifiable space has a largest $e$-compactification $e X$, and he asked whether the space $(e X)_{S}$ - the semiregularization of $e X-$ is always compact. We show that this need not be the case, even if the space $X$ is assumed to be Tychonoff. The example of the space we present is based on an example of $\mathrm{J}$. Chaber.

\section{Preliminary definitions and theorems.}

Definition 2.1 [He]. Let $D$ be a dense subspace of $X$. $X$ is said to be e-compact with respect to $D$ if each open cover of $X$ contains a finite subcollection that covers $D$. If so, $X$ is called an e-compactification of $D$ and $D$ is called e-compactifiable.

Observe that within this terminology the expression "let $X$ be an $e$-compact space" is meaningless. From this definition it readily follows that an e-compactification of a space $X$ is an $H$-closed extension. The following theorem shows that the converse need not be true.

THEOREM $2.2[\mathrm{He}]$. Let $p X$ be an extension of $X$. Then the following statements are equivalent:

(i) $p X$ is an e-compactification of $X$.

(ii) Every ultrafilter on $X$ has an accumulation point in $p X$.

(iii) $p X$ is $H$-closed and $X \cup\{q\}$ is regular, for all $q \in p X$.

It follows that an e-compactifiable space is regular. The converse is not the case. From 2.2(iii) we can conclude that each noncompact $\mathcal{R}$-closed space (i.e. a regular space which is closed in every regular space in which it is embedded, see [BS]) is an example of a regular non-e-compactifiable space. It is clear that every Tychonoff space is e-compactifiable, and in [He] the question appeared whether the converse

Received by the editors February 21, 1983.

1980 Mathematics Subject Classification. Primary 54C10, 54D20, 54G20; Secondary 54D25.

Key words and phrases. e-compactifiable spaces, perfect maps.

c1983 American Mathematical Society $0002-9939 / 83 \$ 1.00+\$ .25$ per page 
holds. In the next section we show that this is not the case. We were unable to characterize the class of $e$-compactifiable spaces in terms of some separation property.

The following properties of $e$-compactifiable spaces are known.

TheOREM 2.3 [He]. (i) Let $p X$ be an e-compactification of $X$. Then $\operatorname{cl}_{p X} Y$ is an e-compactification of $Y$, for each $Y \subset X$.

(ii) Let $p_{i} X_{i}$ be an e-compactification of $X_{i}(i \in I)$. Then $\prod p_{i} X_{i}$ is an e-compactification of $\Pi X_{i}$.

Recall that a subset $U \subset X$ is regular-closed if cl int $U=U$. The collection of regular-closed subsets of $X$ is a closed base for some topology on $X$. $X$ supplied with this topology is called the semiregularization of $X$, to be denoted by $X_{S} . X$ is called semiregular if $X$ is homeomorphic to $X_{S}$.

In [St] R. M. Stephenson observed that Theorem 2.3 implies that each $e$-compactifiable space $X$ has a largest $e$-compactification $e X$, i.e. if $\alpha X$ is an $e$-compactification of $X$ then the map id: $X \rightarrow \alpha X$ has a continuous extension over $e X$.

THEOREM 2.4. (i) [St] Let $X$ be an e-compactifiable space. Then $X$ is an open subspace of $\mathrm{e} X$ and $\mathrm{e} X-X$ is a closed discrete subspace of $e X$.

(ii) Let $f: X \rightarrow Y$ be a continuous map and assume that both $X$ and $Y$ are e-compactifiable. Then there is a continuous extension ef: $e X \rightarrow e Y$ of $f$.

Proof. (ii) According to 2.3(ii) we have that $e X \times e Y$ is an $e$-compactification of $X \times Y$. Define $\tilde{X}=\{(x, f(x)): x \in X\} \subset X \times Y . \tilde{X}$ is a closed subset of $X \times Y$ and $\Pi_{X} \uparrow \tilde{X}: \tilde{X} \rightarrow X$ is a homeomorphism. Since $\operatorname{cl}_{e X \times e_{Y}} \tilde{X}$ is an $e$-compactification of $\tilde{X}$, the map $\left(\Pi_{X} \uparrow \tilde{X}\right)^{-1}: X \rightarrow \tilde{X}$ has an extension $e\left(\prod_{X} \uparrow \tilde{X}\right)^{-1}: e X \rightarrow \mathrm{cl}_{e^{\prime} X \times e^{Y}} \tilde{X}$. Define ef $=\Pi_{e Y} \circ e\left(\Pi_{X} \uparrow \tilde{X}\right)^{-1}$.

As a method to answer the question of S. Hechler, R. M. Stephenson asked the following question.

"Let $X$ be an $e$-compactifiable space. Is the space $(e X)_{S}$ always compact?"

Our example of a non-Tychonoff $e$-compactifiable space provides a negative answer to this question. A partial positive answer to Stephenson's question is the following

Theorem 2.5 [St]. Let $X$ be a regular space. If disjoint regular closed sets are contained in disjoint open subsets (in particular, if $X$ is normal), then $X$ is Tychonoff (hence e-compactifiable) and $(e X)_{S}$ is compact.

Our second example shows that the answer is negative if $X$ is only assumed to be Tychonoff. The following simple lemma is one of the keys to the construction.

LEMMA 2.6. Let $X$ be a Tychonoff space. Then $(e X)_{S}$ is compact iff the map e(id): $e X \rightarrow \beta X$ is injective.

Proof. Observe that $X$ is a subspace of $(e X)_{S}$ and that the map $e(\mathrm{id}):(e X)_{S} \rightarrow \beta X$ is also continuous. Then we have " $\rightarrow$ ", since $(e X)_{S}$ is a compactification of $X$ and " $\leftarrow$ " holds because $(e X)_{S}$ is minimal Hausdorff and the topology of $\beta X$ is weaker than that of $(e X)_{S}$. 
3. The results. The following theorem is the key to our construction of a non-Tychonoff $e$-compactifiable space.

THEOREM 3.1. Perfect preimages of e-compactifiable spaces are e-compactifiable.

Proof. Let $X$ be an $e$-compactifiable space and let $f: Y \rightarrow X$ be a perfect map. We construct an $e$-compactification $\alpha Y$ of $Y$ in the following way. The underlying set of $\alpha Y$ is $Y \oplus(e X-X)$ and a topology is defined by

(i) $Y$ is open in $\alpha Y$;

(ii) For $p \in \alpha Y-Y=e X-X$ the collection $\mathcal{Q}_{p}=\left\{\{p\} \cup f^{-1}(X \cap U): U\right.$ open in $e X \& p \in U\}$ is taken as a local base in $p \in \alpha Y$.

One readily sees that $\alpha Y$ is a Hausdorff extension of $Y$. To see that $\alpha Y$ is an $e$-compactification of $Y$, consider an ultrafilter $\mathscr{F}$ on $Y$. Then $f(\mathscr{F})=\{f(F): F \in \mathscr{F}\}$ is an ultrafilter on $X$; hence $f(\mathscr{F})$ has an accumulation point $q$ in $e X$. If $q \in X$ then, since $f$ is perfect, $\mathscr{F}$ has an accumulation point in $f^{-1}(q)$. If $q \in e X-X$, then $f(F) \cap U_{q} \neq \varnothing$ for each open neighborhood $U_{q}$ of $q$ in $e X$ and $F \in \mathscr{F}$. Since $f(F) \subset X$ it follows that $F \cap f^{-1}\left(U_{q} \cap X\right) \neq \varnothing$, i.e. $q$-considered as an element of $\alpha Y$-is an accumulation point of $\mathscr{F}$.

In [Ch] J. Chaber constructed examples of non-Tychonoff perfect preimages of Tychonoff spaces, and so these examples establish the existence of non-Tychonoff $e$-compactifiable spaces. From 2.3(i) it follows that subspaces of perfect preimages of Tychonoff spaces are $e$-compactifiable. We were not able to construct $e$-compactifiable spaces outside this particular class. Observe that a space $X$ in this class (with $|X|>1)$ admits nonconstant real-valued continuous functions.

Question 3.2. Do there exist $e$-compactifiable spaces on which every real-valued continuous function is constant?

Let us now answer the question of R. M. Stephenson, whether there exist Tychonoff spaces $X$ for which $(e X)_{S}$ is not compact. Our strategy is as follows. We construct a Tychonoff space $X$, a point $p \in \beta X-X$ and an extension $\alpha X$ of $X$ such that $|\alpha X-X|>1$ and such that the map $f: \alpha X \rightarrow X \cup\{p\}(\subset \beta X)$ defined by $f(x)=x \quad(x \in X)$ and $f(\alpha X-X)=p$ is perfect. It then follows that $\alpha X$ is $e$-compactifiable, and since $e \alpha X$ can be considered as an $e$-compactification of $X$, we can conclude from the diagram below that the map $e(\mathrm{id}): e X \rightarrow \beta X$ is not injective. ( $e_{1}$ is the extension of id: $X \rightarrow \alpha X \subset e \alpha X$ to $e X$ (see 2.4(iii)).) ( $e_{2}$ is the extension of id: $X \cup\{p\} \rightarrow \beta(X \cup\{p\})$ to $e(X \cup\{p\}$.) Indeed, the diagram shows that $e(\mathrm{id})=$ $e_{2} \circ$ ef $\circ e_{1}$; hence $e(\mathrm{id})^{-1}(p)>1$.

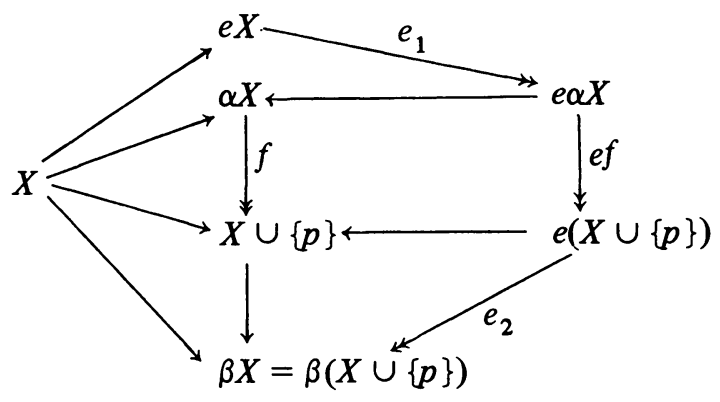


The example we present is almost identical to the one constructed by $\mathrm{J}$. Chaber. The only difference lies in the fact that we want the point $p$ to lie in the Cech-Stone remainder of $X$. For the reader's convenience we give the construction in detail.

Example 3.3. Put $T=\left(\omega_{1}+1\right) \times\left(\omega_{1}+1\right)-\left\{\left(\omega_{1}, \omega_{1}\right)\right\}$. The set of pairs of the form $\left(\alpha, \omega_{1}\right) \in T$ will be called the left edge of $T$. The set of pairs of the form $\left(\omega_{1}, \alpha\right) \in T$ will be called the right edge of $T$. Define the space $T^{n}$, for $n \in \mathbf{N}$, as the space obtained by identification in the sum $\bigoplus_{i=1}^{n} T(i)$ where $T(i)=T \times\{i\}$, of the right edge of $T(i)$ with the left edge of $T(i+1)$. Let $\varphi_{n}: \bigoplus_{i=1}^{n} T(i) \rightarrow T^{n}$ be the corresponding identification-map. For each $0 \leqslant k \leqslant n$ we define an open subset $U_{k}^{\prime \prime} \subset T^{n}$, by

$$
U_{k}^{n}= \begin{cases}\text { int } \varphi_{n}(T(1)) & (k=0), \\ \text { int } \varphi_{n}(T(k) \cup T(k+1)) & (k=1, \ldots, n-1), \\ \text { int } \varphi_{n}(T(n)) & (k=n) .\end{cases}
$$

Finally we define $X=\bigoplus_{n=1}^{\infty} T^{n}$.

It is well known that $\left|\beta T^{n}-T^{n}\right|=1$, for each $n \in \mathbf{N}$. For $\alpha<\omega_{1}$ put $Z_{\alpha}=$ $\left[\alpha, \omega_{1}\right] \times\left[\alpha, \omega_{1}\right]-\left\{\left(\omega_{1}, \omega_{1}\right)\right\}$. Then $\left\{Z_{\alpha}: \alpha<\omega_{1}\right\}$ is a base for the unique nonfixed $z$-ultrafilter on $T$. If we define, for $n \in \mathbf{N}, Z_{\alpha}^{n}=\varphi_{n}\left(\oplus_{i=1}^{n}\left(Z_{\alpha} \times(i)\right)\right)$ then $\left\{Z_{\alpha}^{n}\right.$ : $\left.\alpha<\omega_{1}\right\}$ is a base for the unique nonfixed $z$-ultrafilter $\mathbf{Z}^{n}$ on $T^{n}$.

Next we define a point $p \in \beta X-X$. Let $\mathcal{Q}$ be a nonfixed ultrafilter on $\mathbf{N}$. For $G \in \mathcal{Q}$ and $\alpha<\omega_{1}$ put $Z(G, \alpha)=\cup\left\{Z_{\alpha}^{n}: n \in G\right\}$. It is easy to verify that the collection $\left\{Z(G, \alpha): G \in \mathcal{Q}, \alpha<\omega_{1}\right\}$ is a base for a nonfixed $z$-ultrafilter $(\bar{y}$ on $X$. Let $p \in \beta X-X$ be the point in $\beta X$ corresponding to $\widetilde{F}$, i.e. $\{p\}=\cap\left\{\operatorname{cl}_{\beta X} F: F \in(\bar{T}\}\right.$. In the space $X \cup\{p\}$ we have the following: If $U$ is open in $X$ then $U \cup\{p\}$ is a neighborhood of $p$ in $X \cup\{p\}$ iff $\exists G \in \mathcal{G} \exists \alpha<\omega_{1}$ such that $Z(G, \alpha) \subset U$. (*)

(This is not completely trivial, since $X$ is not normal. However, it follows easily by considering the space $\tilde{X}=\bigoplus_{n=1}^{\infty} \mathrm{cl}_{\beta X} T^{n} \subset \beta X$, which is $\sigma$-compact (hence normal). We omit the details.)

Let us now introduce a topology on the set $X \cup[0,1]([0,1]$ is the unit interval) in the following way. For $t \in[0,1]$ let $\left\{V_{l}(t)\right\}_{l=1}^{\infty}$ be a countable local base at $t$. For $t \in[0,1], l \in \mathbf{N}, G \in \mathcal{G}, \alpha<\omega_{1}$ define

$$
U(t, l, G, \alpha)=\bigcup_{n \in G} \bigcup_{s \in V_{l}(t)}\left(U_{[n . s]}^{n} \cap Z_{\alpha}^{n}\right) \cup V_{l}(t)
$$

(Here $[n . s]$ denctes the greatest integer not greater than $n . s$.$) And next we put:$

$X$ is open in $X \cup[0,1]$.

For $t \in[0,1]$ the collection $\left\{U(t, l, G, \alpha): l \in \mathbf{N}, G \in \mathcal{G}, \alpha<\omega_{1}\right\}$ is defined to be a local base of $t$ in $X \cup[0,1]$.

Observe that $[0,1]$ is embedded in $X \cup[0,1]$. It is easy to check that $X \cup[0,1]$ is a Hausdorff space. In fact our topology has more open sets than Chaber's.

Claim. Let $U$ be a subset of $X \cup[0,1]$ which contains [0,1]. Then $U$ is neighborhood of $[0,1]$ in $X \cup[0,1]$ iff $\exists G \in \mathcal{G} \exists \alpha<\omega_{1}$ such that $Z(G, \alpha) \subset U$. 
Proof. Assume $Z(G, \alpha) \subset U$. Then, for $t \in[0,1], t \in U(t, l, G, \alpha) \subset Z(G, \alpha)$. Hence $[0,1] \subset$ int $U$. On the other hand, assume $[0,1] \subset$ int $U$. Then, $\forall t \in[0,1]$ $\exists l(\mathrm{t}) \in \mathbf{N} \exists G(t) \in \mathcal{Q} \exists \alpha(t)<\omega_{1}$ such that

$$
t \in U(t, l(t), G(t), \alpha(t)) \subset U .
$$

Since $[0,1]$ is compact, $[0,1]$ can be covered by finitely many of these sets. Say $[0,1] \subset \cup_{i=1}^{k} U\left(t_{i}, l\left(t_{i}\right), G\left(t_{i}\right), \alpha\left(t_{i}\right)\right)(\subset U)$. Put $G=\bigcap_{i=1}^{k} G\left(t_{i}\right)(\in \mathcal{G})$ and $\alpha=$ $\sup \left\{\alpha\left(t_{i}\right): i \leqslant k\right\} \quad\left(<\omega_{1}\right)$. We claim that $Z(G, \alpha) \subset \cup_{i=1}^{k} U\left(t_{i}, l\left(t_{i}\right), G\left(t_{i}\right), \alpha\left(t_{i}\right)\right)$ $(\subset U)$. Choose $p \in Z(G, \alpha)$, say $p \in Z_{\alpha}^{n}$ for some $n \in G$. Since $T^{n}=\cup_{k=0}^{n} U_{k}^{n}$, there exists $k \leqslant n$ such that $p \in U_{k}^{n}$. Choose $s \in[0,1]$ such that $[n . s]=k$. If $s \in U\left(t_{i}, l\left(t_{i}\right), G\left(t_{i}\right), \alpha\left(t_{i}\right)\right)$ then, since $G \subset G\left(t_{i}\right)$ and $Z_{\alpha} \subset Z_{\alpha\left(t_{1}\right)}$, we conclude that $p \in Z\left(\alpha\left(t_{i}\right)\right) \cap U_{[n . s]}^{n}$ for "some" $n \in G\left(t_{i}\right)$, i.e. $p \in U\left(t_{i}, l\left(t_{i}\right), G\left(t_{i}\right), \alpha\left(t_{i}\right)\right)$. The claim follows.

From the claim and from $(*)$ we conclude that the space obtained from $X \cup[0,1]$ by identifying $[0,1]$ to a point is homeomorphic to $X \cup\{p\}$. Obviously the map $f$ : $X \cup[0,1] \rightarrow X \cup\{p\}$ defined by $f(x)=x(x \in X)$ and $f[0,1]=p$ is a perfect map. Hence, all the required properties are satisfied.

REMARK 3.4. It is well known that each space $T^{n}$, as defined in 3.3 , has a unique (nontrivial) regular extension, namely $\beta T^{n}$. It follows that $\mathrm{cl}_{e X} T^{n} \simeq \beta T^{n}$, for all $n \in \mathbf{N}$. Consider the space $\tilde{X}=\bigoplus_{n=1}^{\infty} \beta T^{n}$. Then $X \subset \tilde{X} \subset e X . \tilde{X}$ is a $\sigma$-compact, hence normal, and according to 2.5 this implies that $(e \tilde{X})_{S}=\beta \tilde{X}=\beta X$. Since $(e X)_{S} \neq \beta X$, we conclude that the map id: $\tilde{X} \rightarrow e X$ cannot be extended continuously to $e \tilde{X}$. At first glance this may seem a contradiction, but it is not. One cannot use 2.4(ii) to ensure that such an extension should exist since $e X$ is not $e$-compactifiable ( $e X$ is not even semiregular), nor the fact that $e \tilde{X}$ is the largest $e$-compactification, since $e X$ is not an $e$-compactification of $\tilde{X}$. (A nonfixed ultrafilter on $\tilde{X}-X$ does not have an accumulation point in $e X$.)

\section{REFERENCES}

[BS] M. P. Berri and R. H. Sorgenfrey, Minimal regular spaces, Proc. Amer. Math. Soc. 14 (1963), 454-458.

[Ch] J. Chaber, Remarks on open-closed mappings, Fund. Math. 73 (1971), 197-208.

[He] S. H. Hechler, On a notion of weak compactness in non-regular spaces, Studies in Topology (N. M. Stavrakas and K. R. Allen, eds.), Academic Press, New York, 1975, pp. 215-237.

[St] R. M. Stephenson, Not every minimal Hausdorff space is e-compact, Proc. Amer. Math. Soc. 52 (1975), 381-388.

Subfaculteit Wiskunde en Informatica, Vrije Universiteit, De BoelelaAn 1081, 1081 HV Amsterdam, The Netherlands (Current address of K. P. Hart)

Current address (J. Vermeer): Department of Mathematics, University of Kansas, Lawrence, Kansas 66045 